\title{
I quarant'anni del Museo Monumento al deportato di Carpi
}

L'8 dicembre 2013, il Museo Monumento al deportato politico e razziale di Carpi ha festeggiato il quarantennale dall'inaugurazione, riaprendo al pubblico dopo un intervento di manutenzione profonda. Un museo che oggi, a quarant'anni dalla sua inaugurazione e addirittura a cinquanta dalla sua ideazione, ha uno straordinario valore di documento, testimoniando una precocissima fase di «museografia della memoria».

December 8th, 2013, the Museum Monument to the political and racial deportee of Carpi (Modena) commemorated the 40th anniversary from its inauguration day with a new opening after a careful conservation. Today, after forty years from its original opening and even fifty from its conception, this museum has a extraordinary value as document, testifying a very early phase of the "museography of memory».

L'8 dicembre 2013, con un poco di ritardo rispetto all'anniversario preciso, il Museo Monumento al deportato politico e razziale di Carpi ha festeggiato il quarantennale dall'inaugurazione, riaprendo al pubblico dopo un intervento di manutenzione profonda. Il ripristino degli intonaci, del colore delle incisioni graffiate su di essi e della pavimentazione, laddove danneggiata, è stato accompagnato anche da un accurato lavoro sul contenuto delle teche, riportate alla disposizione iniziale degli oggetti grazie alla collaborazione con Anna Steiner, figlia dei grafici e architetti Albe e Lica che curarono il progetto del museo insieme agli architetti BBPR, e del suo archivio familiare.

Un museo che oggi, a quarant' anni dalla sua inaugurazione e addirittura a cinquanta dalla sua ideazione, ha uno straordinario valore di documento, testimoniando 


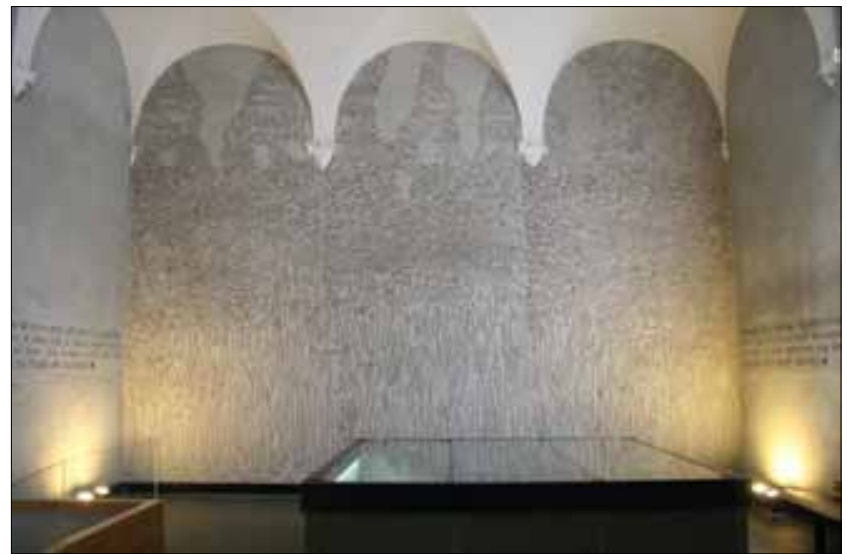

Museo Monumento al deportato politico e razziale, graffito di Alberto Longoni una precocissima fase di «museografia della memoria». Una fase in cui la ricerca storica non era ancora approfondita e i paradigmi retorici affermati non erano in grado di cogliere a fondo la complessità del sistema concentrazionario, ma allo stesso tempo gli autori - molto spesso coinvolti in prima persona negli eventi della guerra e della deportazione - mettevano in gioco le proprie competenze progettuali, creative e professionali per un imperativo etico, prima ancora che per finalità informative, conoscitive o didattiche.

\section{Carpi ricorda il campo di Fossoli}

Il 14 ottobre 1973 la grande piazza della piccola cittadina di Carpi si riempì di persone, assiepate sotto gli ombrelli, come testimoniano le fotografie dell'epoca. Accanto ai carpigiani, delegazioni provenienti da altre città italiane ed europee e da Israele: l'occasione per l'afflusso di oltre 40.000 persone era data dall'inaugurazione del Museo Monumento al deportato politico e razziale all'interno del Palazzo dei Pio.

Il motivo per cui quel complesso memoriale era stato realizzato proprio nella cittadina della provincia modenese risiedeva nella vicinanza con il campo di Fossoli, punto fondamentale della mappa della deportazione italiana. Creato nel maggio 1942 per l'internamento di prigionieri di guerra, dopo l'8 settembre 1943 passò in gestione alla Repubblica sociale, diventando, alla fine dell'anno, un campo di concentramento per ebrei e politici. All'inizio del 1944 subentrò il comando nazista di Verona e il campo venne suddiviso in due zone, una sotto controllo italiano e una sotto la giurisdizione tedesca. In questa amministrazione mista, gli italiani controllavano gli internati civili (in molti casi cittadini di paesi nemici), mentre il campo nuovo gestito dai tedeschi divenne un Durchgangslager, un campo di transito per ebrei e politici, destinati all'ulteriore trasporto verso campi ben più terribilmente famosi come Auschwitz, Bergen-Belsen, Ravensbrück, Mau- 


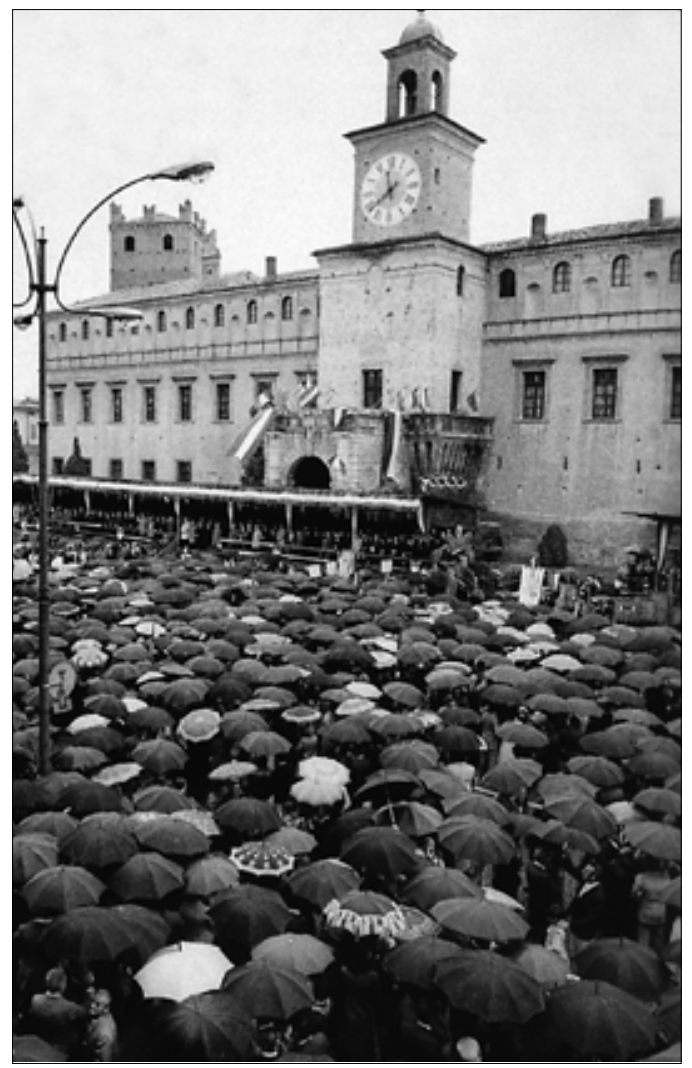

Inaugurazione, Carpi 1973

thausen. La scelta di utilizzare un campo proprio in questa località della pianura emiliana aveva avuto ragioni strategiche, ovvero il suo posizionamento lungo la linea ferroviaria del Brennero.

Il campo italiano fu dismesso per ordine del Ministero dell'Interno della Rsi a metà luglio 1944, mentre gli ultimi convogli in partenza dal campo tedesco datano all'agosto; nei mesi successivi l'avanzata alleata costrinse il comando del Sicherheitsdienst (il servizio per la sicurezza del Reich) di Verona a spostare le funzioni del campo a Bolzano-Gries. Ma il campo di Fossoli non rimase vuoto: continuò a essere operativo fino al novembre 1944 nella raccolta di lavoratori coatti da convogliare verso la Germania.

Non è facile calcolare il numero dei deportati passati attraverso il campo di Fossoli: le ricerche storiche sono arrivate a indicare circa 5.000 prigionieri di guerra nel primo anno, circa 2.500-3.000 politici - la cui meta finale fu spesso Mauthausen e il suo sistema di sottocampi -, cui si aggiungono, tra il febbraio e l'agosto 1944, circa un terzo degli 8.000 ebrei deportati dall'Italia.

A conclusione della guerra al campo viene cambiata destinazione, adeguandola alle urgenze di quel periodo di spostamenti, in cui tutto il corpo europeo era attraversato da displaced persons di ritorno dalla guerra e dai campi, che tentavano di raggiungere la propria casa o si muovevano con la prospettiva ancora più disarmante di doverne cercare una nuova. È allora che Fossoli diventa un campo per indesiderabili, stranieri che nella primavera del 1945 si trovano nel territorio italiano (criminali di guerra che non vogliono rientrare nel proprio paese temendo persecuzioni, ex-combattenti in disaccordo con i regimi sorti nelle loro patrie dopo il conflitto, donne, orfani, sbandati, ecc.). Nelle baracche di Fossoli vengono riuniti per essere rimpatriati oppure, come nel caso di molti ebrei, si raccolgo- 
no in attesa di raggiungere un porto da cui salpare verso la Palestina.

È nel 1947 che il campo vive una radicale trasformazione: diventa la sede di Nomadelfia, comunità voluta da don Zeno Saltini per occuparsi di ragazzi orfani di guerra, fondata sui precetti evangelici e il lavoro della terra. Viene cambiato di segno al senso del luogo: da spazio di coercizione e dolore a spazio per la rinascita. Questo mutamento funzionale lascia tracce anche sulle baracche che, tramite interventi materiali per migliorarne le condizioni (isolamento contro l'umidità e il freddo) divengono case e laboratori.

Cinque anni dopo ancora un passaggio d'uso, che testimonia uno dei complessi nodi critici derivati dalla fine del conflitto: 1'ex campo diventa luogo di accoglienza per i profughi giuliano-dalmati, venendo così ribattezzato Villaggio San Marco. Agli interventi dei nomadelfi per rendere accettabile la vita all'interno delle ex baracche, si aggiungono le migliorie di questa fase, volte a renderle più accoglienti, con intonaci colorati e siepi curate. Molti vi resteranno quasi vent'anni, fino all'inizio degli anni Settanta, quando il campo viene definitivamente abbandonato.

\section{La prima mostra dei Lager nazisti e il progetto di un Museo Monumento}

La presenza del campo aveva coinvolto la vita della comunità carpigiana già durante gli anni della guerra, pur senza avere consapevolezza del ruolo che il campo ricopriva all'interno del ramificato e capillare sistema concentrazionario nazista, un ruolo per nulla marginale nella «logistica» italiana della deportazione.

Già nei primi anni Cinquanta l'amministrazione di Carpi, guidata dal sindaco Bruno Losi (antifascista fortemente attivo nella Resistenza) si pone il problema di come ricordare quanto accaduto, come riuscire a elaborarlo non solo a livello locale, ma propriamente nazionale. Nel decennale della conclusione del conflitto viene così organizzata all'interno del Palazzo dei Pio la prima mostra fotografica dei Lager nazisti: l'esposizione presentava, riprodotte e attaccate semplicemente su piccoli pannelli di cartone, centinaia di fotografie dei Lager, scattate prevalentemente dagli Alleati, e dai fotografi che seguirono la loro avanzata, all'apertura dei campi tedeschi. Immagini ben note ora, ma che allora, dieci anni dopo i fatti, lasciavano sgomenti, soprattutto raccolte in quella quantità ed esposte tutte insieme: quell'orrore era appena accaduto, toccava da vicino molti dei visitatori. Era cronaca, non storia.

Il nome di Fossoli, in quella mostra, veniva affiancato ai più noti e famigerati campi tedeschi e il suo ruolo nel sistema della deportazione era testimoniato 
dalle fotografie degli anni della guerra: il luogo, infatti, in quello stesso periodo ospitava gli esuli istriano-dalmati, con tutte le alterazioni delle strutture originarie che questa trasformazione d'uso aveva comportato. L'idea di poter rendere il luogo stesso uno spazio per il ricordo e la riflessione pubblica era forse ancora prematura e il destino di riuso funzionale del campo di Fossoli era condiviso da molte strutture analoghe sparse per l'Europa, per lo più riutilizzate prima per i prigionieri tedeschi e successivamente per i profughi; solo nel caso di AuschwitzBirkenau si era proceduto immediatamente alla trasformazione del campo in un monumento, in un monito per la memoria, attraverso un progetto museale che, tuttavia, generò alterazioni delle strutture del Lager.

A Fossoli, allora Villaggio San Marco, l'amministrazione carpigiana intervenne alla fine delle celebrazioni per il decennale, inaugurando un monumento sobrio ed essenziale: un muro di pietre con inserite all'interno un'urna contenente terra proveniente da alcuni campi nazisti e una lapide con incisa un'epigrafe di Piero Calamandrei.

L'idea di realizzare a Carpi un segno forte e permanente in ricordo della deportazione italiana prende corpo durante le celebrazioni per il Centenario dell'Unità d'Italia, quando Losi - divenuto presidente di un comitato formato dai rappresentanti degli enti locali (Comuni di Carpi e Modena, Provincia di Modena), dall'Associazione nazionale ex deportati-Aned, dall'Unione delle comunità ebraiche, da varie associazioni di antifascisti e ex partigiani come l'Associazione nazionale partigiani d'Italia-Anpi e l'Associazione nazionale perseguitati politici italiani antifascisti-Anppia e dall'Istituto storico della Resistenza di Modena - pronuncia in Parlamento un discorso volto a sensibilizzare i rappresentanti politici e ottenere il loro sostegno.

Nel corso del 1962 viene così elaborato un bando di concorso per il Museo Monumento al deportato politico e razziale, da realizzare nelle sale del Palazzo dei Pio, che viene pubblicato nel gennaio successivo.

Il testo del bando esprime molto chiaramente la volontà di incenti-

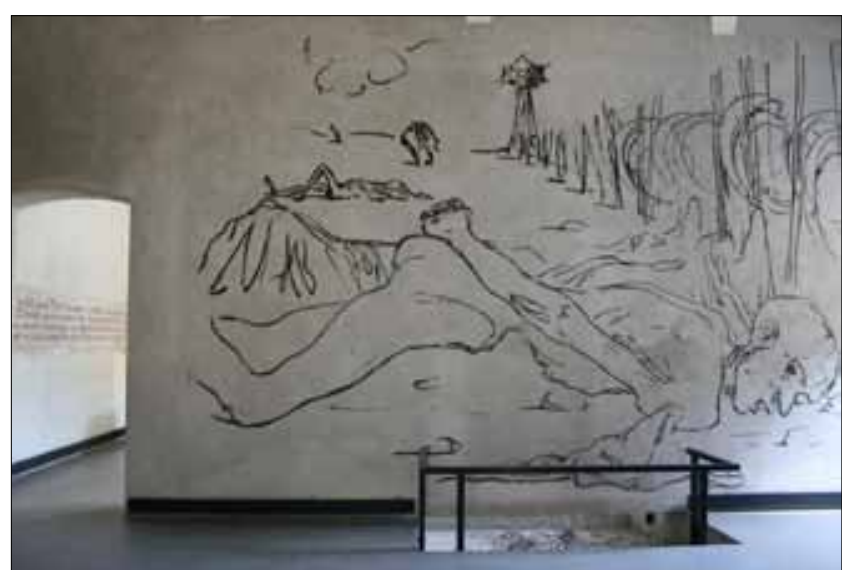

Museo Monumento al deportato politico e razziale, graffito di Corrado Cagli 
vare la partecipazione e la collaborazione: l'unione tra le diverse associazioni di resistenti e deportati deve trovare un riflesso nella collaborazione del mondo della progettazione con quello dell'arte, come, ad esempio, era già avvenuto per il memoriale delle Fosse Ardeatine. La coralità deve essere il carattere principale del progetto.

\section{Trasformare l'esperienza in progetto: il museo dei BBPR}

Il progetto vincitore è quello del gruppo di architetti BBPR - Gian Luigi Banfi, Lodovico Barbiano di Belgiojoso, Enrico Peressutti ed Ernesto Nathan Rogers: già attivi negli anni del fascismo, nel dopoguerra sono tra i protagonisti di un'importante e raffinata stagione progettuale della museografia italiana. Ma, soprattutto, il loro lavoro del dopoguerra rivolge una forte attenzione ai progetti in ricordo della deportazione, dato che due dei suoi componenti - Belgiojoso e Banfi - erano stati deportati a Gusen come prigionieri politici: se il primo riesce a fare ritorno, il secondo vi muore nell'aprile del 1945.

Il progetto per il museo carpigiano è così qualcosa di profondamente legato all'esperienza degli stessi architetti, cui si affiancano Albe e Lica Steiner, coinvolti per il reperimento del materiale iconografico e la sua elaborazione, e Nelo Risi che individua i passi dalle Lettere dei condannati a morte della Resistenza europea (Einaudi, Torino 1954) da incidere sulle pareti del museo, insieme alle riproduzioni di un'opera di Renato Guttuso, degli schizzi realizzati da Corrado Cagli dopo l'apertura dei cancelli di Buchenwald e di un lavoro di Alberto Longoni, ritornato dalla prigionia come internato militare italiano-Imi, oltre che di disegni di Picasso e Léger. La tecnica dello «spolvero», un'incisione quasi graffiata dell'intonaco fresco, viene effettuata dalla Cooperativa muratori di Carpi, in modo da fare emergere lo strato sottostante di intonaco color «sangue rappreso» oppure nero. La parte centrale delle sale viene occupata da poche e rarefatte teche che mostrano pochi e scelti oggetti, accompagnati da immagini fotografiche riprodotte a grandi dimensioni e trattate graficamente con sgranature e retini.

La natura del progetto risiede in questa estrema semplicità che si traduce in incisività: le pareti spoglie, da cui emergono le parole dei prigionieri e i graffiti che, nel caso di Cagli e Longoni, sono essi stessi testimonianze. Nelle teche, pochi oggetti, carichi del valore di reliquie, come le posate e la matricola personali di Belgiojoso e le lettere di Mino Steiner, donate dal fratello Albe: tutti gli oggetti hanno un valore prima di tutto personale, di cose sopravvissute. La narrazione che si dipana attraverso il percorso ha un tono estremamente secco: è un'emozione composta quella che deve guidare nella visita, lontana dalla «spettacolarizzazione» che 


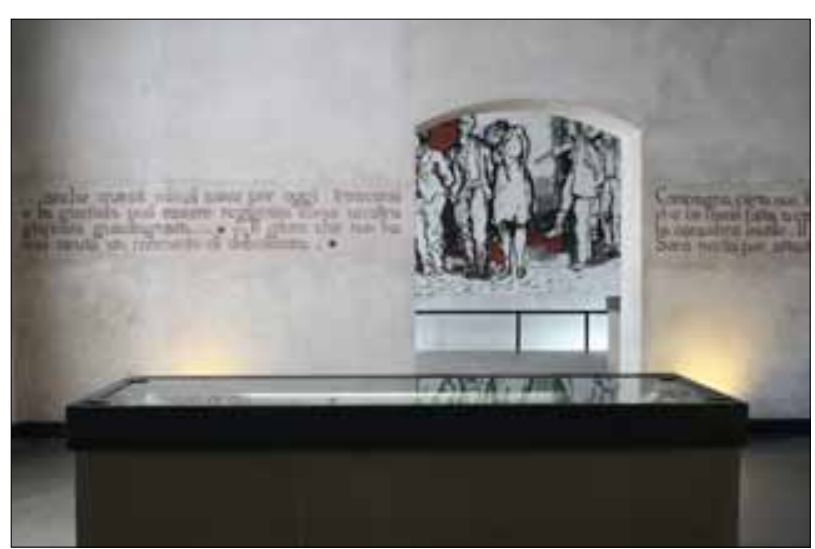

Museo Monumento al deportato politico e razziale, sala e teca, sul fondo graffito di Renato Guttuso (particolare)

impronterà molti musei della memoria degli anni Ottanta e Novanta Il valore del Museo, oggi, a quarant'anni di distanza, è prima di tutto quello di documento di una stagione in cui gli stessi testimoni dei fatti partecipavano in prima persona alla «costruzione» dei segni memoriali. Un'epoca in cui la memoria della deportazione era improntata principalmente al «paradigma resistenziale» e allo stesso tempo era comunicata in forma diretta ed evocativa, senza apparati di natura didattica. Nei decenni successivi, la ricerca ha messo in luce come la lettura politica della deportazione non potesse bastare a renderne un'adeguata memoria (e tornano in mente le prime pagine de La tregua, in cui Primo Levi racconta la differente percezione della liberazione da parte propria e di un anziano deportato politico tedesco, Thylle).

In questo panorama, il Museo Monumento carpigiano ha cercato precocemente di raccontare le varie componenti della deportazione - politici, discriminati razziali, Imi - seppur all'interno della cornice del proprio presente (fortemente caratterizzata in senso politico), elaborando una forma che si distingue tuttora per raffinatezza, sobrietà e incisività del disegno museale.

\section{Bibliografia}

Di Sante C. 2008, Il campo per gli "indesiderabili”. Documenti e immagini del "Centro raccolta profughi stranieri” di Fossoli (1945-1947), Torino EGA

Di Sante C. 2011, Stranieri indesiderabili. Il campo di Fossoli e i "centri raccolta profughi" in Italia (1945-1970), Verona: Ombre Corte

Iezzi R. 2007/2008, Storia e memoria della deportazione nel museo. Alcuni casi esempla$r i$, tesi di laurea specialistica in Storia dell'arte, Università di Bologna

Gibertoni R., Melodi A. 1993, Il Museo Monumento al Deportato a Carpi, Milano: Electa. Leoni G. (ed.) 1990, Trentacinque progetti per Fossoli, Milano: Electa 
Luppi M., Ruffini E. (eds.) 2005, Immagini dal silenzio. La prima mostra nazionale dei Lager nazisti attraverso l'Italia 1955-1960, Carpi: Nuovagrafica

Mira R. 2010, Il campo di Fossoli come centro di raccolta di manodopera: agosto-novembre 1944, in Tromboni 2010, pp. 107-119

Molinari M.L. 2006, Storia di un villaggio per profughi giuliani, Torino: EGA

Montanari M. (ed.) 2003, Architetture della memoria. Ideazione, progettazione, realizzazione del Museo monumento al deportato di Carpi, Reggio Emilia: Grafitalia

Ori A.M. 2004, Il Campo di Fossoli. Da luogo di prigionia e deportazione a luogo di memoria 1942-2004, Carpi: APM

Picciotto L. 2010, L'alba ci colse come un tradimento. Gli ebrei nel campo di Fossoli 1943-1944, Milano: Mondadori

Ruffini E. 2006, Un lapsus di Primo Levi. Il testimone e la ragazzina, Bergamo: Assessorato alla cultura/Istituto bergamasco per la storia della Resistenza e dell'età contemporanea - Isrec

Silingardi C. 2010, Prigionieri e internati a Fossoli (Modena) 1942-1944, in Tromboni 2010, pp. 121-132

Tibaldi I. 1994, Compagni di viaggio. Dall'Italia ai lager nazisti: i trasporti dei deportati, 1943-1945, Milano: Franco Angeli

Tromboni D. (ed.) 2010, Storie di esilio, di fuga e di deportazione. Ferraresi ed emilianoromagnoli nella diaspora ebraica e antifascista, atti della giornata di studi, Ferrara: Tresogni

\section{Risorse}

Sito Fondazione ex campo di Fossoli

http://www.fondazionefossoli.org

Video testimonianza di Anna Steiner realizzata l'8 dicembre 2013 in occasione delle celebrazioni per il quarantesimo anniversario del Museo Monumento al deportato di Carpi http://www.youtube.com/watch?v=uDLUeeX5b6I

Trailer del film-documentario Crocevia Fossoli, regia di Federico Baracchi e Roberto Zampa 2013, realizzato dalla Fondazione ex campo di Fossoli http://www.youtube.com/watch?v=u69JqONhJqE 